Lasha Ephremidze, A. Razmadze Mathematical Institute, Georgian Academy of Sciences, Tbilisi 380093, Georgia. e-mail: Lasha@rmi.acnet.ge

\title{
ON REVERSE WEAK $(1,1)$ TYPE INEQUALITIES FOR MAXIMAL OPERATORS WITH RESPECT TO ARBITRARY MEASURES
}

\begin{abstract}
Necessary and sufficient conditions on a measure are obtained for the corresponding maximal operators to be of reverse weak $(1,1)$ type.
\end{abstract}

Let $\nu$ be a locally finite non-negative Borel measure on the real line $\mathbb{R}$. For any locally integrable (with respect to $\nu$ ) function $f, f \in L_{\mathrm{loc}}(\nu)$, the maximal functions $M_{\nu}^{+}(f)$ and $M_{\nu}(f)$ are defined by

$$
\begin{aligned}
& M_{\nu}^{+}(f)(x)=\sup _{x<b, \nu[x, b)>0} \frac{1}{\nu[x, b)} \int_{[x, b)}|f| d \nu, \\
& M_{\nu}(f)(x)=\sup _{a<x<b, \nu(a, b)>0} \frac{1}{\nu(a, b)} \int_{(a, b)}|f| d \nu, \quad x \in \mathbb{R} .
\end{aligned}
$$

An operator $T: L_{\mathrm{loc}}(\nu) \rightarrow L_{0}(\mathbb{R})$ (The latter notation stands for the class of measurable functions.) is said to be of (locally) reverse weak $(1,1)$ type if there exists an independent constant $C$ such that

$$
\nu\left\{\left(T\left(\chi_{I} f\right)>\lambda\right) \cap I\right\} \geq \frac{1}{\lambda \cdot C} \int_{\left(T\left(\chi_{I} f\right)>\lambda\right) \cap I}|f| d \nu
$$

for every $f \in L_{\mathrm{loc}}(\nu)$ and interval $I=(\alpha, \beta)$, whenever $\lambda>\max \left(T\left(\chi_{I} f\right)(\alpha)\right.$, $\left.T\left(\chi_{I} f\right)(\beta)\right)$.

That the maximal operators $M_{\nu}$ and $M_{\nu}^{+}$are of reverse weak $(1,1)$ type when $\nu$ is the Lebesgue measure was proved in [1], [2]. It is also well-known that in general $M_{\nu}$ and $M_{\nu}^{+}$may not be of this type. Theorems 1 and 2 below give necessary and sufficient conditions on the measure $\nu$ for the corresponding maximal operators to be of reverse $(1,1)$ type.

Key Words: Maximal functions, reverse weak type inequality.

Mathematical Reviews subject classification: 42B25

Received by the editors November 17,1998 
Theorem 1. There exists a constant $C$ such that

$$
\nu\left\{\left(M_{\nu}^{+}\left(\chi_{I} f\right)>\lambda\right) \cap I\right\} \geq \frac{1}{\lambda \cdot C} \int_{\left(M_{\nu}^{+}\left(\chi_{I} f\right)>\lambda\right) \cap I}|f| d \nu
$$

for every $f \in L_{\mathrm{loc}}(\nu)$ and $I=(\alpha, \beta)$ whenever

$$
\lambda>M_{\nu}^{+}\left(\chi_{I} f\right)(\alpha)
$$

if and only if

$$
\sup _{\nu(a, b)>0} \frac{\nu[a, b)}{\nu(a, b)} \leq C .
$$

For any $f \in L_{\mathrm{loc}}(\nu)$, if $x \in\left(M_{\nu}^{+}(f)>\lambda\right) \equiv G_{\lambda}^{+}$, then there is $\delta_{x}>0$ such that $y \in G_{\lambda}^{+}$for each $y \in\left(x-\delta_{x}, x\right]$. Thus the connected components of $G_{\lambda}^{+}$ will necessarily be the intervals open from the left.

We need the following Lemma.

Lemma. Let $f \in L_{\mathrm{loc}}(\nu)$. If an interval $] a, b \mid$ is a connected component of $G_{\lambda}^{+}$(the sign $\mid$next to $b$ indicates that $b$ either belongs or does not belong to $G_{\lambda}^{+}$), then $\left.\nu\right] a, b \mid>0$.

Proof. If $b \in G_{\lambda}^{+}$, i.e. $] a, b \mid=(a, b]$, then there exists a sequence $b_{n}, n=$ $1,2, \ldots$, from $\mathbb{R} \backslash G_{\lambda}^{+}$which tends to $b$ from the right. Assuming that $b^{\prime}$ is a number greater than $b$ for which $\frac{1}{\nu\left[b, b^{\prime}\right)} \int_{\left[b, b^{\prime}\right)}|f| d \nu>\lambda$, we will get

$$
\frac{1}{\nu\left[b_{n}, b^{\prime}\right)} \int_{\left[b_{n}, b^{\prime}\right)}|f| d \nu \nrightarrow \frac{1}{\nu\left[b, b^{\prime}\right)} \int_{\left[b, b^{\prime}\right)}|f| d \nu
$$

Thus $\nu\{b\}>0$.

If $b \notin G_{\lambda}^{+}$, then we can consider any $x \in(a, b)$. Since we know that there exists $x^{\prime}>x$ such that

$$
\int_{\left[x, x^{\prime}\right)}|f| d v>\lambda \nu\left[x, x^{\prime}\right)
$$

and $\int_{\left[b, x^{\prime}\right)}|f| d v \leq \lambda \nu\left[b, x^{\prime}\right)$ whenever $x^{\prime}>b$, we can conclude that (4) holds for some $x^{\prime} \in(a, b]$. Hence $\nu(a, b)>0$.

Proof of Theorem 1. Suppose there exists a constant $C>1$ such that (3) holds. Assume $f \in L_{\mathrm{loc}}(\nu), I=(\alpha, \beta)$ and $\lambda$ is sufficiently large so that inequality (2) holds. Since $(-\infty, \alpha] \cap\left(M_{\nu}^{+}\left(\chi_{I} f\right)>\lambda\right)=\varnothing$, to prove inequality (1) it is sufficient to show that

$$
\nu] a, b\left|\geq \frac{1}{C \lambda} \int_{] a, b \mid}\right| f \mid d \nu
$$


holds, where $] a, b \mid$ is a connected component of $\left(M_{\nu}^{+}\left(\chi_{I} f\right)>\lambda\right) \equiv G_{\lambda}^{+}\left(\chi_{I} f\right)$. Indeed,

$$
\nu\left[a, b\left|\geq \frac{1}{\lambda} \int_{[a, b \mid}\right| f \mid d \nu,\right.
$$

since $a \notin G_{\lambda}^{+}\left(\chi_{I} f\right)$, and by virtue of the Lemma and inequality (3) we have $\nu] a, b \mid>0$ and

$$
\nu[a, b \mid \leq C \nu] a, b \mid .
$$

Hence (6) and (7) imply (5). The sufficient part of the theorem is proved.

If $(a, b)$ is an interval such that $\nu(a, b)>0$ and $\frac{\nu[a, b)}{\nu(a, b)}>C$ for some constant $C>1$, then we can take $f=\chi_{(a, b)}$. We will have

$$
M_{\nu}^{+}(f)(a)=\sup _{a<x \leq b} \frac{\nu(a, x)}{\nu[a, x)}=\frac{\nu(a, b)}{\nu[a, b)} .
$$

So

$$
M_{\nu}^{+}(f)(x) \leq M_{\nu}^{+}(f)(a)<\frac{1}{C},
$$

when $x \leq a$, and $M_{\nu}^{+}(f)(x)=1$ when $a<x<b$ and $\nu[x, b)>0$. Thus for each $\lambda \in\left(M_{\nu}^{+}(f)(a), \frac{1}{C}\right)$ we have

$$
\nu\left(M_{\nu}^{+}(f)>\lambda\right)=\nu(a, b)=\int_{(|f|>\lambda)} f d \nu,
$$

and inequality (1) fails to hold ( $I$ is assumed to be $(a, b))$.

Remark. Theorem 1 asserts that if $\nu$ has some atom and $M_{\nu}^{+}$is of reverse weak $(1,1)$ type, then starting from this point $\nu$ necessarily consists of isolated atoms.

Theorem 2. There exists a constant $C$ such that

$$
\nu\left\{\left(M_{\nu}\left(\chi_{I} f\right)>\lambda\right) \cap I\right\} \geq \frac{1}{\lambda \cdot C} \int_{\left(M_{\nu}\left(\chi_{I} f\right)>\lambda\right) \cap I}|f| d \nu
$$

for every $f \in L_{\mathrm{loc}}(\nu)$ and $I=(\alpha, \beta)$ whenever

$$
\lambda>\max \left(M_{\nu}\left(\chi_{I} f\right)(\alpha), M_{\nu}\left(\chi_{I} f\right)(\beta)\right)
$$

if and only if

$$
\sup _{\nu(a, b)>0} \min \left(\frac{\nu[a, b)}{\nu(a, b)}, \frac{\nu(a, b]}{\nu(a, b)}\right) \leq C .
$$


Proof. Clearly, for the operator $M_{\nu}$ the set $\left(M_{\nu}(f)>\lambda\right) \equiv G_{\lambda}$ is now open and similarly to the Lemma

$$
\nu(a, b)>0
$$

if $(a, b)$ is a connected component of $G_{\lambda}$. Suppose there exists a constant $C>1$ such that (10) holds. Assume $f \in L_{\mathrm{loc}}(\nu), I=(\alpha, \beta)$ and $\lambda$ is sufficiently large so that inequality (9) holds. Just as in Theorem 1, to prove inequality (8) it is sufficient to show that

$$
\nu(a, b) \geq \frac{1}{C \lambda} \int_{(a, b)}|f| d \nu,
$$

where $(a, b)$ is a connected component of $\left(M_{\nu}\left(\chi_{I} f\right)>\lambda\right)$. Since $M_{\nu}\left(\chi_{I} f\right)(x) \leq$ $\lambda$ for $x=a, b$, we readily have

$$
\nu[a, b) \geq \frac{1}{\lambda} \int_{(a, b)}|f| d \nu, \quad \nu(a, b] \geq \frac{1}{\lambda} \int_{(a, b)}|f| d \nu .
$$

It follows from (10) and (13) that (12) holds.

If now $(a, b)$ is an interval such that (11) holds and

$$
\min \left(\frac{\nu[a, b)}{\nu(a, b)}, \frac{\nu(a, b]}{\nu(a, b)}\right)>C>1,
$$

then one can consider the function $f=\chi_{(a, b)}$ just like in Theorem 1. We have

$$
M_{\nu}(f)(a)=\frac{\nu(a, b)}{\nu[a, b)} \quad \text { and } \quad M_{\nu}(f)(b)=\frac{\nu(a, b)}{\nu(a, b]} .
$$

Obviously, $M_{\nu}(f)(x) \leq \min \left(M_{\nu}(f)(a), M_{\nu}(f)(b)\right)$ when $x \in \mathbb{R} \backslash(a, b)$ and $M_{\nu}(f)(x)=1$ when $x \in(a, b)$. Thus for each $\lambda \in\left(\max , \frac{1}{C}\right)$ we have

$$
\nu\left(M_{\nu}(f)>\lambda\right)=\nu(a, b)=\int_{(|f|>\lambda)} f d \nu
$$

and inequality (8) fails to hold.

\section{References}

[1] E. M. Stein, A Note on the class L $\log L$. Studia Math. 33(1969), 305-310.

[2] O. D. Tsereteli, On the inversion of some Hardy-Littlewood theorems. (Russian) Soobshch. Akad. Nauk Gruz. SSR 56(1969), No. 2, 269-271. 